\title{
Adult Versus Pluripotent Stem Cell-Derived Mesenchymal Stem Cells: The Need for More Precise Nomenclature
}

\author{
Michael D. West ${ }^{1} \cdot$ Igor Nasonkin $^{1} \cdot$ David Larocca $^{2} \cdot$ Karen B. Chapman $^{3}$. \\ Francois Binette $^{1,4} \cdot$ Hal Sternberg ${ }^{1}$
}

Published online: 28 July 2016

(C) The Author(s) 2016. This article is published with open access at Springerlink.com

\begin{abstract}
The complexity of human pluripotent stem cell (hPSC) fate represents both opportunity and challenge. In theory, all somatic cell types can be differentiated from hPSCs, opening the door to many opportunities in transplant medicine. However, such clinical applications require high standards of purity and identity, that challenge many existing protocols. This underscores the need for increasing precision in the description of cell identity during hPSC differentiation. We highlight one salient example, namely, the numerous published reports of hPSC-derived mesenchymal stem cells (MSCs). We suggest that many of these reports likely represent an improper use of
\end{abstract}

This article is part of the Topical Collection on Stem Cells: Policies from the Bench to the Clinic

Michael D. West

mwest@biotimemail.com

Igor Nasonkin

inasonkin@biotimemail.com

David Larocca

dlarocca@biotimemail.com

Karen B. Chapman

kchapman@oncocyte.com

Francois Binette

fbinette@biotimemail.com

Hal Sternberg

hsternberg@biotimemail.com

1 BioTime, Inc., 1010 Atlantic Ave., Alameda, CA 94501, USA

2 ReCyte Therapeutics, 1010 Atlantic Ave, Alameda, CA 94501, USA

3 OncoCyte Corporation, 1010 Atlantic Ave, Alameda, CA 94501, USA

4 OrthoCyte Corporation, 1010 Atlantic Ave, Alameda, CA 94501, USA certain cluster of differentiation (CD) antigens in defining bone marrow-derived MSCs. Instead, most such hPSC-derived mesenchymal cells are likely a complex mixture of embryonic anlagen, primarily of diverse mesodermal and neural crest origins, making precise identification, reproducible manufacture, and uniform differentiation difficult to achieve. We describe a potential path forward that may provide more precision in nomenclature, and cells with higher purity and identity for potential therapeutic use.

Keywords Embryonic stem cells · Mesenchymal stem cells · Clonal embryonic progenitor cells $\cdot$ Neural crest $\cdot$ Cartilage . Bone

$\begin{array}{ll}\text { Abbreviations } \\ \text { CFU-EP } & \text { Colony-forming unit-embryonic progenitor } \\ \text { CFU-F } & \text { Colony-forming unit-fibroblast } \\ \text { CNS } & \text { Central nervous system } \\ \text { FACS } & \text { Fluorescence activated cell sorting } \\ \text { GMP } & \text { Good manufacturing practices } \\ \text { hEP cells } & \text { Human embryonic progenitor cells } \\ \text { hES cells } & \text { Human embryonic stem cells } \\ \text { hPSCs } & \text { Human pluripotent stem cells } \\ \text { LMD } & \text { LifeMap Discovery } \\ \text { MSCs } & \text { Mesenchymal stem cells } \\ \text { NMF } & \text { Non-negative matrix factorization } \\ \text { RFUs } & \text { Relative fluorescence units }\end{array}$

\section{Introduction}

Human pluripotent stem cells (hPSCs), including such cell types as human embryonic stem cell (hESC) lines from biparental or parthenogenetic blastocysts or comparable cells 
produced by nuclear transfer or induced pluripotent stem cell (iPSC) technology, are being widely utilized for basic research and a small number of human therapeutic trials. In addition to pluripotency, hPSCs have the unique property of unlimited proliferation through the abundant expression of the catalytic component of telomerase that generally maintains them with long and stable telomere length [1]. Since telomere length predicts the replicative capacity of cultured somatic cells [2], hPSCs are seen as a renewable source of diverse human somatic cell types with long proliferative lifespan potential.

In contrast to hPSCs, adult stem cells, such as hematopoietic stem cells (HSCs), neuronal stem cells (NSCs), and mesenchymal stem cells (MSCs), generally possess limited differentiation potential (multipotency) and a finite replicative capacity. This limited scale-up potential commonly leads to the need for continual sourcing of tissue to derive new product lots. In the case of MSCs, there is also a loss of differentiation potential after $5-12$ passages in vitro $[3,4]$. These challenges have, therefore, led to a search for means of manufacturing the counterparts of these adult stem cell types from hPSCs [5].

\section{Identity of Adult-Derived MSCs}

Bone marrow is the site of definitive hematopoiesis in the adult human. Within the bone marrow are cell subpopulations including blood cell progenitors as well as stromal cells $[6,7]$. The stromal cells, designated "mesenchymal stem cells" by Arnold Caplan, have been widely studied primarily for their potential utility in the repair of osteochondral tissues [8], though exploratory investigations have also used them for such diverse applications as in the treatment of diabetes [9], heart disease [10], CNS disorders [11], as well as others. Despite numerous attempts to improve terminology and resolve confusion regarding the similarities and differences of putative MSCs from diverse tissue sites, the term continues to be used for any stromal cell isolate that shows evidence of differentiation to osteochondral and adipose cell fates and displays a defined set of cell surface antigens. The original rationale for referring to all such populations as MSCs is that, by definition, stem cells must be able to self-renew and to differentiate. Thus, if stromal cells from a particular tissue can expand in culture (i.e., self-renew) and differentiate down the osteochondral and adipose lineages, then they were referred to as MSCs. This terminology, however, may prevent recognition of important differences in such cells when derived from diverse tissue types such as adipose, umbilical cord, and bone marrow.

To illumine the rationale behind the use of commonly used antigens in identifying MSCs, it is helpful to recall some of the history of early hematology. Since the bone marrow is the site of definitive hematopoiesis in the adult, early efforts to characterize the progenitors of diverse blood cell lineages such as lymphoid (B and $\mathrm{T}$ cells), myeloid (monocytes and granulocytes), and erythroid cells (such as red cell progenitors) utilized cell cloning through colony-forming unit (CFU) assays performed in tissues or in methylcellulose similar to those previously used to isolate and characterize bacterial strains on agar. These blood colony-forming unit assays together with other assays led to the identification of cluster of differentiation (CD) antigens that allowed the lineages to be precisely identified. One such useful antigen was CD45 (lymphocyte common antigen) that was observed to be common to all leucocytes [12]. In contrast to blood cell progenitors, the stromal cells that propagated in cultures from the bone marrow were designated colony-forming unit-fibroblasts (CFU-F). Since CD antigens were an active area of research at the time, $\mathrm{CD}$ antigen profiles were sought to distinguish the CFU-F from the blood cell types. CFU-F cells were observed to be CD45- [13] and express antigens not normally expressed on blood cell progenitors such as CD29, CD73, CD90, and CD105 [14, 15]. The International Society for Cellular Therapy has suggested that the term "MSC," or as they suggest, "multipotent mesenchymal stromal cells," be applied to cells that express the abovementioned pattern of CD antigens, can be cultivated in adherent cultures, and show the potential to differentiate into at least osteochondral and adipocyte lineages [16].

\section{Identity of hPSC-Derived Mesenchymal Cells}

A growing number of reports suggest that MSCs can be robustly generated from hPSCs $[17,18]$. The markers used are generally the same as those used to identify adult-derived cells; however, the reported differentiation protocols vary widely [19]. Although these pluripotent stem cell-derived "MSCs" share classically-defined MSC surface markers such as CD29, CD90, CD73, and CD105, and show some similarities in gene expression profiles, they also display clear functional and expression profile differences that distinguish them from adult MSCs [18]. Given that there are potentially thousands of distinct cell types that can be derived from hPSCs, it is reasonable to inquire whether the putative hPSC-derived MSCs actually correspond to adult-derived MSCs or represent a variety of different, as yet unidentified, embryonic progenitor cell types.

We previously performed a colony-forming unit analysis on human embryonic progenitor (hEP) cells derived from $\mathrm{hESC}$ in adherent culture to more precisely identify the various types of progenitor cells that constitute hPSC-derived mesenchyme. $\mathrm{hEP}$ cells are cultures of hPSCs-derived cells that have lost markers of pluripotency, but have yet to terminally differentiate. We used transcriptomic analysis of global gene expression data to provide an objective measure of the complexity of a hEP cell library of 202 clones. We performed non-negative matrix factorization (NMF) analysis [20] of the data which revealed that there were 140 distinct cell types contained within the library (stability score; $k=140$ ). As evidence of the 
accuracy of this complexity analysis, the NMF clustering did not split biological or technical replicates, and individual clones showed diverse site-specific homeobox gene markers as well as markers of diverse types of mesoderm, such as lateral plate, intermediate, and paraxial mesoderm, as well as diverse types of neural crest cells corresponding to the NMF clustering.

Of these 140 clonal hEP cell lines, seven have been characterized as cells that, like MSCs, can robustly differentiate into osteochondral lineages as indicated by COL2A1 expression when differentiated in micromass culture in the presence of TGF $\beta 3[21 \bullet \cdot]$. We compared typical MSC marker gene expression in a panel of randomly selected clonal hEP lines, a group of adult-derived MSC lines, and a panel of numerous diverse adult epithelial, mesenchymal, and blood cell types. Analysis of $C D 29, C D 73, C D 90$, and $C D 105$ gene expression showed that these genes were not only expressed by MSCs, but also by most adult-derived cells including such diverse cell types as endothelial cells, urothelial and retinal pigment epithelial cells, Schwann cells, diverse fibroblast types, and smooth muscle cells from multiple tissues. Originally, these markers were chosen to distinguish MSCs from non-adherent hematopoietic cells in the bone marrow and as expected, they were largely absent from a diverse panel of blood cell types. $C D 45$, a marker of hematopoietic cells, however, was expressed only in blood cell types and not in the other cell types tested. Similar patterns of CD antigen expression were also observed by flow cytometry. Therefore, it is reasonable to conclude that the expression of the markers $C D 29, C D 73$, $C D 90$, and $C D 105$, and the absence of $C D 45$ expression, are effective markers in distinguishing bone marrow stromal cells from blood cells, but are ineffective in distinguishing MSCs from most other somatic cell types.

Our clonal analysis reveals that while expressing commonlyused MSC markers, only a subset of hPSC-derived embryonic mesenchymal cell types are capable of differentiating into osteochondral (COL2A1 and IBSP-expressing) [21••] and adipogenic (FABP4-expressing) cells [22•, 23]. It is, therefore, reasonable to conclude that reports that hPSC-derived heterogeneous cultures of mesenchymal cells are "MSCs" is likely
CD29
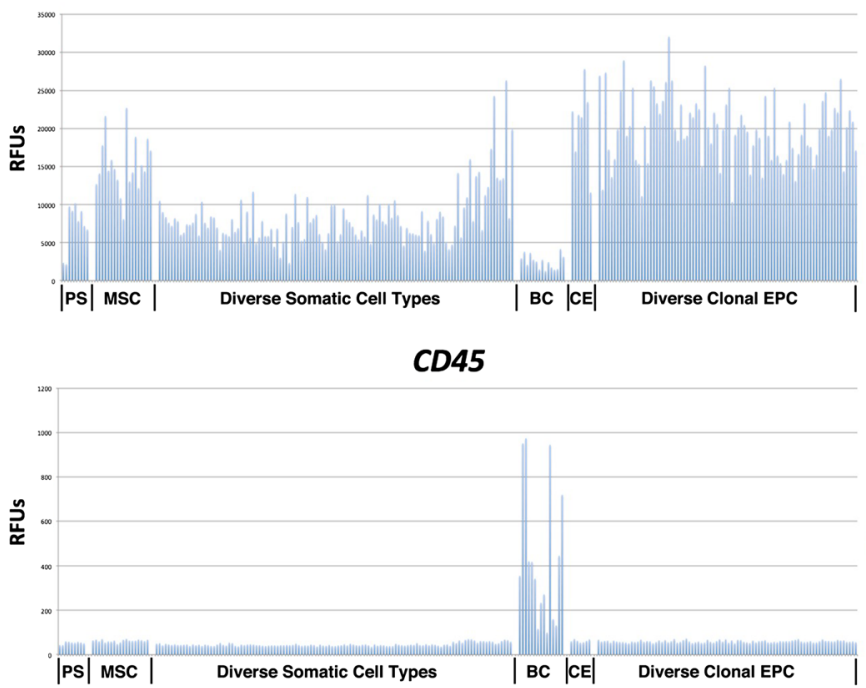

CD73

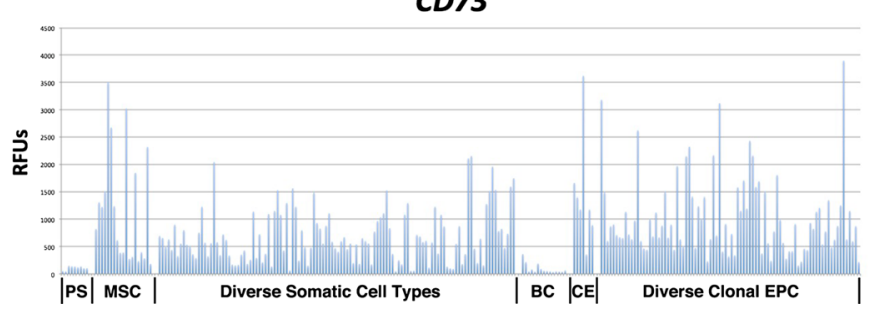

Fig. 1 Expression levels of common MSC CD antigen markers in hPSC, adult stromal, blood, and embryonic progenitor cell types. The levels of expression (in relative fluorescence units $(R F U s)$ ) of the marker genes $C D 29, C D 45, C D 73, C D 74, C D 90$, and $C D 105$ are shown. $P S$ human pluripotent (embryonic) stem cells; $M S C$ adult bone marrow-derived mesenchymal stem cells, diverse somatic cell types 111 fetal or adult-
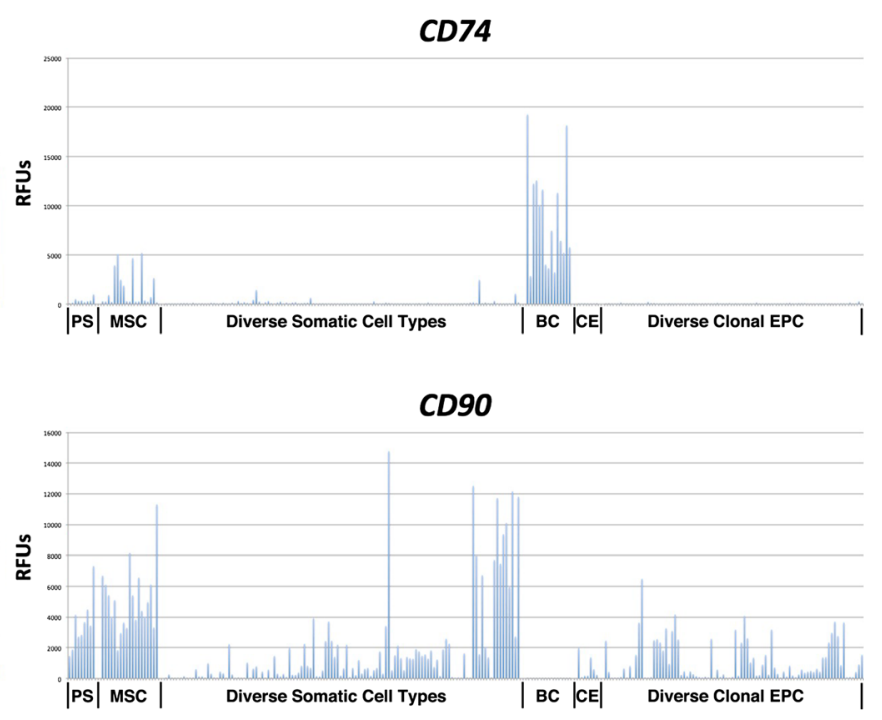

CD105

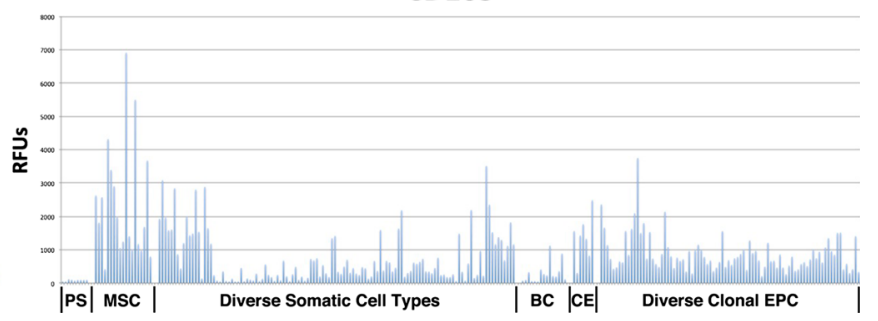

derived epithelial and mesenchymal cell types, $B C 15$ diverse blood cell types, $C E$ seven hPSC-derived clonal embryonic progenitor cell lines previously shown to have osteochondral differentiation potential similar to adult MSCs, and 86 other diverse clonal embryonic progenitor (EPC) lines (used with permission from Regen. Med. (2013) 8(2), 125-144) $[21 \cdot]$ 
an imprecise and unhelpful use of terminology. Instead, they are a complex mixture of cell types, many of which may express commonly-used MSC markers, but others with very distinct cellular identities and/or cell fate. In such heterogeneous cultures, a subset of the cells capable of expressing adipogenic or chondrogenic markers in the presence of BMPs like TGF $\beta 3$ could lead to the incorrect conclusion that the entire MSC marker expressing population had similar potential.

It has been reported that the antigen CD74 is capable of distinguishing bone marrow MSCs from other types of mesenchymal cells [24]. As shown in Fig. 1, CD74 is indeed expressed on an RNA level in definitive MSCs, but largely absent from the diverse hPSC-derived hEP cell lines, even those with a robust potential for chondrogenic differentiation. Given the lack of $C D 74$ expression in the majority of the clonal isolates, it appears likely that many published reports of MSCs derived from hPSCs should not be considered as meeting the most stringent criteria of true MSCs unless, at minimum, they were shown to express CD74. Many of these still-uncharacterized embryonic mesenchymal cell types may indeed have cellular fates and functionality that distinguishes them from MSCs. For example, we observed that clonal EP cell lines capable of osteochondral fates differentiate into cartilage lacking markers of hypertrophy such as COL1OAI and IHH [25-27], the former being the most abundant transcript in adult bone marrow-derived MSCs and a marker of cells destined to promote vascular invasion rather than forming definitive chondrocytes such as those residing in the hyaline articular cartilage [28]. In this context, hPSC-derived EP lines (that do not differentiate to hypertrophic chondrocytes) may be better therapeutic candidates than MSCs (which are capable of differentiating to hypertrophic chondrocytes) for repairing damaged joints with cartilage having the needed mechanical properties for weight bearing joints. In addition, given the typically long initial telomere length of many hPS cells, the proliferative lifespan while retaining differentiation potential of the hEP cell lines can exceed that of adult-derived MSCs [25]. Therefore, we suggest that future studies of hPSC-derived mesenchymal cells should explore the unique and diverse properties of embryonic anlagen rather than assuming they are functionally identical to adult-derived MSCs. In addition, we suggest that, unless a precise association with specific anlagen such as forelimb bud mesenchyme or mandibular neural crest mesenchyme can be established, the hPSC-derived heterogeneous mesenchymal cultures be designated as "human embryonic fibroblasts," instead of MSCs, in the same way that heterogeneous mouse embryo-derived mesenchyme is referred to as mouse embryonic fibroblasts (MEFs) and not MSCs.

\section{Conclusion}

The complexity of the differentiated fate space of human pluripotent stem cells challenges current differentiation protocols.
This is particularly the case when the intended use is for human clinical application. The demonstration that many heterogeneous cultures of mesenchymal cells from hPSC contains a diversity of up to 140 -fold diversity out of 202 clonal lineages, combined with the recognition that many of these cell types have very different fates than bone marrow MSCs such as documented neural crest lineages capable of meningeal and choroid plexus differentiation, should cause concern when the intent is to generate the equivalent of adult-derived MSCs for human transplantation [5]. The hPSC-derived counterparts may, therefore, have very different fates, efficacy, and safety profiles.

It seems likely, therefore, that future trends in the field will need to include new and improved differentiation protocols that yield a higher resolution of the definition of the precise cell types being produced as well as where in the time course of embryological development the cells most closely correspond. Also critical, in our opinion, will be a global map of the transcriptome of human development with a cellular level of resolution. This cell ontology tree, similar to that being built at LifeMap Discovery [29] (discovery.lifemapsc.com), when properly correlated with the corresponding transcriptome of each cell type, may lead to a deeper understanding of the complex cascade of molecular development as well as a means of reproducibly manufacturing more precisely defined cellular therapeutics.

Acknowledgment Drs. Caplan and Bonfield wish to thank Dr. Benjamin Goldstein for his kind assistance in the reviewing of this article.

\section{Compliance with Ethical Standards}

Conflict of Interest MDW, HS, and IN, are employees of BioTime, Inc. (Alameda, CA). MDW is employed by BioTime, Inc., and receives stock options (from BioTime, Inc., OrthoCyte Corporation, ReCyte Therapeutics, and OncoCyte Corporation) as a result of his employment. He has over 100 patents and patent applications worldwide in the field of pluripotent stem cell technology; a list of relevant patents and patent applications is listed on www.michaelwest.org. DL is an employee of ReCyte Therapeutics (Alameda, CA), has received grant support from CIRM, and has been issues a patent (patent \# 9175263) for methods to identify and enrich hPSC progenitor cell lines. HS is an employee of BioTime, Inc. (Alameda, CA), has received grant support from CIRM, has been issued a patent (patent \# 8685386), and has pending patents (application \# 13/683,241, application \# 14/131,429, and application \# $14 / 048,910$ ). $\mathrm{KBC}$ is an employee of OncoCyte Corporation (Alameda, $\mathrm{CA}$ ). FB is an employee of OrthoCyte Corporation (Alameda, CA). ReCyte Therapeutics, OncoCyte Corporation, and OrthoCyte Corporation are subsidiaries of BioTime, Inc. (Alameda, CA), and LifeMap Discovery is a database operated by LifeMap Sciences, Ltd, a subsidiary of BioTime.

Human and Animal Rights and Informed Consent This article does not contain any studies with human or animal subjects performed by any of the authors.

Open Access This article is distributed under the terms of the Creative Commons Attribution 4.0 International License (http:// 
creativecommons.org/licenses/by/4.0/), which permits unrestricted use, distribution, and reproduction in any medium, provided you give appropriate credit to the original author(s) and the source, provide a link to the Creative Commons license, and indicate if changes were made.

\section{References}

Papers of particular interest, published recently, have been highlighted as:

- Of importance

•- Of major importance

1. Thomson JA et al. Embryonic stem cell lines derived from human blastocysts. Science. 1998;282:1145-7.

2. Allsopp RC et al. Telomere length predicts replicative capacity of human fibroblasts. Proc Natl Acad Sci U S A. 1992;89:10114-8.

3. Izadpanah $\mathrm{R}$ et al. Long-term in vitro expansion alters the biology of adult mesenchymal stem cells. Cancer Res. 2008;68:4229-38.

4. Madeira A et al. Human mesenchymal stem cell expression program upon extended ex-vivo cultivation, as revealed by 2-DEbased quantitative proteomics. PLoS One. 2012;7:e43523.

5. Lian Q et al. Derivation of clinically compliant MSCs from CD105+, CD24- differentiated human ESCs. Stem Cells. 2007;25:425-36.

6. Friedenstein AJ, Chailakhjan RK, Lalykina KS. The development of fibroblast colonies in monolayer cultures of guinea-pig bone marrow and spleen cells. Cell Tissue Kinet. 1970;3:393-403.

7. Friedenstein AJ, Petrakova KV, Kurolesova AI, Frolova GP. Heterotopic of bone marrow. Analysis of precursor cells for osteogenic and hematopoietic tissues. Transplantation. 1968;6:230-47.

8. Caplan AI. Mesenchymal stem cells. Journal of Orthopaedic Research: Official Publication of The Orthopaedic Research Society. 1991;9:641-50.

9. Li L, et al. Transplantation of mesenchymal stem cells improves type 1 diabetes mellitus. Cell and Tissue Research. 2015;364:34555.

10. Qayyum AA, Mathiasen AB, Kastrup J. Stem cell therapy to treat heart ischaemia: implications for diabetes cardiovascular complications. Curr Diab Rep. 2014;14:554.

11. Redjal N, Zhu Y, Shah K. Combination of systemic chemotherapy with local stem cell delivered S-TRAIL in resected brain tumors. Stem Cells. 2015;33:101-10.

12. Altin JG, Sloan EK. The role of CD45 and CD45-associated molecules in T cell activation. Immunol Cell Biol. 1997;75:430-45.

13. Pittenger MF et al. Multilineage potential of adult human mesenchymal stem cells. Science. 1999;284:143-7.

14. Kobolak J, Dinnyes A, Memic A, Khademhosseini A, Mobasheri A. Mesenchymal stem cells: Identification, phenotypic characterization, biological properties and potential for regenerative medicine through biomaterial micro-engineering of their niche. Methods. 2015;99:62-8

15. Boxall SA, Jones E. Markers for characterization of bone marrow multipotential stromal cells. Stem Cells Int. 2012;2012:975871.

16. Dominici $\mathrm{M}$ et al. Minimal criteria for defining multipotent mesenchymal stromal cells. The International Society for Cellular Therapy position statement. Cytotherapy. 2006;8:315-7.

17. Barberi T, Willis LM, Socci ND, Studer L. Derivation of multipotent mesenchymal precursors from human embryonic stem cells. PLoS Med. 2005;2:e161.

18. Diederichs S, Tuan RS. Functional comparison of human-induced pluripotent stem cell-derived mesenchymal cells and bone marrowderived mesenchymal stromal cells from the same donor. Stem Cells Dev. 2014;23:1594-610.

19. Kimbrel EA et al. Mesenchymal stem cell population derived from human pluripotent stem cells displays potent immunomodulatory and therapeutic properties. Stem Cells Dev. 2014;23:1611-24.

20. West MD et al. The ACTCellerate initiative: large-scale combinatorial cloning of novel human embryonic stem cell derivatives. Regen Med. 2008;3:287-308.

21.• Sternberg H et al. Seven diverse human embryonic stem cellderived chondrogenic clonal embryonic progenitor cell lines display site-specific cell fates. Regen Med. 2013;8:125-44. This study reports on the initial characterization of seven diverse clonal embryonic progenitor cell lines derived from human embryonic stem cells and contrasts their potential for differentiation with adult-derived MSCs.

22. Sternberg $\mathrm{H}$ et al. Human embryonic stem cell-derived neural crest cells capable of expressing markers of osteochondral or meningealchoroid plexus differentiation. Regen Med. 2014;9:53-66. This study shows markers and initial characterization of neural crest lines generated from human embryonic stem cells.

23. Sternberg H, Janus J, West MD. Defining cell-matrix combination products in the era of pluripotency. Biomatter. 2013;3(1):e24496.

24. Ishii $\mathrm{M}$ et al. Molecular markers distinguish bone marrow mesenchymal stem cells from fibroblasts. Biochem Biophys Res Commun. 2005;332:297-303.

25. Sternberg $\mathrm{H}$ et al. A human embryonic stem cell-derived clonal progenitor cell line with chondrogenic potential and markers of craniofacial mesenchyme. Regen Med. 2012;7:481-501.

26. Gebhard $\mathrm{S}$ et al. BAC constructs in transgenic reporter mouse lines control efficient and specific LacZ expression in hypertrophic chondrocytes under the complete Col10a1 promoter. Histochem Cell Biol. 2007;127:183-94.

27. Mak KK, Kronenberg HM, Chuang PT, Mackem S, Yang Y. Indian hedgehog signals independently of PTHrP to promote chondrocyte hypertrophy. Development. 2008;135:1947-56.

28. Pelttari K et al. Premature induction of hypertrophy during in vitro chondrogenesis of human mesenchymal stem cells correlates with calcification and vascular invasion after ectopic transplantation in SCID mice. Arthritis Rheum. 2006;54:3254-66.

29. Edgar R et al. LifeMap Discovery: the embryonic development, stem cells, and regenerative medicine research portal. PLoS One. 2013;8:e66629. 